\title{
Heterotic String Dynamics in the Solvable Lie Algebra Gauge
}

\author{
Nejat T. Yilmaz \\ Department of Mathematics and Computer Science, \\ Çankaya University, \\ Öğretmenler Cad. No:14, 06530, \\ Balgat, Ankara, Turkey. \\ ntyilmaz@cankaya.edu.tr
}

December 24, 2018

\begin{abstract}
A revision of the torodial Kaluza-Klein compactification of the massless sector of the $E_{8} \times E_{8}$ heterotic string is given. Under the solvable Lie algebra gauge the dynamics of the $O(p, q) /(O(p) \times O(q))$ symmetric space sigma model which is coupled to a dilaton, $N$ abelian gauge fields and the Chern-Simons type field strength is studied in a general formalism. The results are used to derive the bosonic matter field equations of the massless sector of the $D$-dimensional compactified $E_{8} \times E_{8}$ heterotic string.
\end{abstract}

\section{Introduction}

The supergravity theory which has the highest spacetime dimension is the $D=11, \mathcal{N}=1$ supergravity [1]. There are three types of supergravity 
theories in ten dimensions namely the IIA [2, 3, 4, the IIB [5, 6, 7] and as the third supergravity, the ten dimensional $\mathcal{N}=1$ type I supergravity theory which is coupled to the Yang-Mills theory [8, 9]. One can obtain the $D=10$, IIA supergravity theory by the Kaluza-Klein dimensional reduction of the $D=11$ supergravity on the circle, $S^{1}$. The supergravity theories in $D<10$ dimensions can be obtained from the $D=11$ and the $D=10$ supergravities by the dimensional reduction and the truncation of fields. A general treatment of the supergravity theories can be found in [10, 11, 12, 13].

The ten dimensional IIA supergravity and the IIB supergravity theories are the massless sectors or the low energy effective limits of the type IIA and the type IIB superstring theories respectively [14]. The type I supergravity theory in ten dimensions on the other hand is the low energy effective limit of the type I superstring theory and the heterotic superstring theory [14]. The eleven dimensional supergravity is conjectured to be the low energy effective limit of the eleven dimensional M-theory.

The symmetries of the supergravity theories are important to understand the symmetries and the duality transformations of the string theories. Especially the global symmetries of the supergravities give the non-perturbative U-duality symmetries of the string theories and the M-theory [15, 16]. An appropriate restriction of the global symmetry group $G$ of the supergravity theory to the integers $\mathbb{Z}$, namely $G(\mathbb{Z})$, is conjectured to be the U-duality symmetry of the relative string theory which unifies the T-duality and the S-duality [14, 15].

In supergravity theories which possess scalar fields, the global (rigid) symmetries of the scalar sector can be extended to the other fields as well, thus the global symmetry of the scalars will be the global symmetry of the entire bosonic sector of the theory. For the majority of the supergravities, the scalar manifolds are homogeneous symmetric spaces which are in the form of cosets $G / K$ [17, and the scalar lagrangians can be formulated as the non-linear coset sigma models, in particular the symmetric space sigma 
models. The dimension of the coset space $G / K$ is equal to the number of the coset scalars of the theory. In general $G$ is a real form of a non-compact semisimple Lie group and $K$ is its maximal compact subgroup. Thus the coset $G / K$ can locally be parameterized through the exponential map by using the solvable Lie subalgebra of $G$ [18. This parametrization is an effective tool for the dynamics of the supergravity theories and it is called the solvable Lie algebra gauge [17].

When we apply the Kaluza-Klein dimensional reduction to the bosonic sector of the $D=11$ supergravity [1] over the tori $T^{n}$, where $n=11-D$, we obtain the $D$-dimensional maximal supergravity theories [19, 20, 21]. The global (rigid) symmetry groups of the bosonic sectors of the reduced lagrangians are in split real form (maximally non-compact). In other words for the scalar coset manifolds, $G / K$ of the maximal supergravities, $G$ which is the global symmetry group, is a semi-simple split real form and $K$ is its maximal compact subgroup. Thus the coset spaces $G / K$ can be parameterized by the Borel gauge which is a special case of the solvable Lie algebra gauge. Therefore the scalar sectors of the maximal supergravities can be formulated as symmetric space sigma models. However we should remark that in certain dimensions in order to formulate the scalar sectors as symmetric space sigma models one has to make use of the dualisation methods to replace the higher-order fields with the newly defined scalars. On the other hand when one considers the Kaluza-Klein compactification of the bosonic sector of the ten dimensional $\mathcal{N}=1$ simple supergravity that is coupled to $N$ abelian gauge multiplets on the tori $T^{10-D}[8,9$, one can show that when a single scalar is decoupled from the others, the rest of the scalars of the lower, $D$-dimensional theories can be formulated as $G / K$ symmetric space sigma models [22]. One can even enlarge the coset formulations of the scalars by using partial dualisations. Unlike the maximal supergravities for this class of supergravities, the global symmetry group $G$ is not necessarily a split real form but it is in general a semi-simple, non-compact real form and again $K$ 
is a maximal compact subgroup of $G$. Therefore in this case one makes use of the more general solvable Lie algebra gauge [17] to parameterize the scalar coset manifolds for the reduced theories.

In this work considering the achievements of [22] we focus on the dynamics of the toroidally compactified low energy effective $E_{8} \times E_{8}$ heterotic string in the solvable Lie algebra gauge. In accordance with the formulation of [22] we will first show how one can obtain a lower dimensional bosonic lagrangian starting from the ten dimensional $\mathcal{N}=1$ type I supergravity theory which is coupled to the abelian Yang-Mills theory [8, 9]. We will formulate the $D$-dimensional bosonic lagrangian in a compact form in which the terms governing the scalars and the coupling abelian gauge fields are constructed in the solvable Lie algebra gauge. Then in a general formalism we will work out in detail the bosonic field equations of the symmetric space sigma model which is coupled to a dilaton, an arbitrary number of abelian gauge fields and a two-form field whose kinetic term is expressed in terms of its Chern-Simons field strength. Our formulation will be a generalized one which uses arbitrary coupling constants. Also it is performed for a general, $O(p, q)$ global symmetry group of the scalar coset. In our generalized formulation we will make use of the construction of [23] to derive the field equations of the coset scalars. To denote the relevance of our construction with the supergravity theories we will give three examples of Maxwell-Einstein supergravities on which the derivation we perform is applicable and whose bosonic field equations correspond to the ones we derive in algebraic terms for the solvable Lie algebra gauge of the scalar coset manifold. Finally we will apply our general results to the case of the $D$-dimensional compactified $E_{8} \times E_{8}$ heterotic string to derive its bosonic matter field equations which show a degree of complexity due to the coupling between the coset scalars and the abelian gauge fields. We will also discuss the relation between different coset parametrizations. Besides we will reckon the correspondence of the two equivalent formulations of the symmetric space sigma model with couplings which are based on different 
scalar coset representations.

In section two we give a brief review of the Kaluza-Klein reduction of the ten dimensional $\mathcal{N}=1$ supergravity that is coupled to the abelian Yang-Mills theory [8, 9] which is studied in detail in [22]. The main objective of section two will be the construction of the $D$-dimensional bosonic lagrangian in a compact form by using the solvable Lie algebra gauge. In section three again under the solvable Lie algebra gauge we will derive the field equations of the symmetric space sigma model which is generally coupled to a dilaton, abelian gauge fields and a Chern-Simons type field strength. In addition we will mention about three examples on which the results can be applied. In the last section we will write down the bosnonic matter field equations of the $D$-dimensional toroidally compactified heterotic string by using the results obtained in section three. We will also discuss the possible field transformations between the two equivalent formulations of the bosonic lagrangian of the compactified heterotic string.

\section{Toroidally Compactified Heterotic String}

In this section we will focus on the Kaluza-Klein reduction on the Euclidean torus $T^{10-D}$ of the bosonic sector of the ten dimensional simple $\mathcal{N}=1$ supergravity [8, 9] which is coupled to $N$ abelian gauge multiplets. We will not cover the details of the reduction steps. A detailed study of the reduction can be referred in [22] in which the structure of the scalar cosets and the matter couplings which appear in each dimension upon the dimensional reduction of the basic fields of the ten dimensional theory are given in detail. When as a special case, the number of the $U(1)$ gauge fields is chosen to be 16 , the ten dimensional supergravity which is coupled to 16 abelian $U(1)$ gauge multiplets becomes the low energy effective limit of the $E_{8} \times E_{8}$ ten dimensional heterotic string theory which forms the massless background coupling [14]. Thus when $N=16$ our formulation corresponds to the dimensional re- 
duction of the low energy effective bosonic lagrangian of the ten dimensional $E_{8} \times E_{8}$ heterotic superstring theory. For $N=16$, the $D=10$ Yang-Mills supergravity [8, 9] has the $E_{8} \times E_{8}$ Yang-Mills gauge symmetry, however the general Higgs vacuum structure causes a spontaneous symmetry breakdown so that the full symmetry $E_{8} \times E_{8}$ can be broken down to its maximal torus subgroup $U(1)^{16}$, whose Lie algebra is the Cartan subalgebra of $E_{8} \times E_{8}$. Thus the ten dimensional Yang-Mills supergravity reduces to its maximal torus subtheory which is an abelian (Maxwell-Einstein) supergravity theory. The bosonic sector of this abelian Yang-Mills supergravity corresponds to the low energy effective theory of the bosonic sector of the fully Higgsed ten dimensional $E_{8} \times E_{8}$ heterotic string theory [14, 22]. Therefore our main concern will be the abelian Yang-Mills supergravity in ten dimensions, however for the purpose of generality we will consider the coupling of $N U(1)$ gauge field multiplets to the graviton multiplet.

As we have discussed before the scalar sectors of the lower dimensional theories can be formulated as non-linear sigma models, more specifically as the symmetric space sigma models. One needs to apply the method of dualisation for some of the fields in certain dimensions to construct the scalar cosets as symmetric space sigma models. The scalar cosets $G / K$ are based on the global internal symmetry groups $G$ which are in general non-compact real forms of semi-simple Lie groups. In supergravity theories the global symmetry of the scalar lagrangian can be extended to the entire bosonic sector of the theory. Under certain conditions the global internal symmetry groups may be maximally non-compact (split) real forms but in general they are elements of a bigger class of Lie groups which contains the global internal symmetry groups of the maximal supergravities namely the split real forms as a special subset. The main difference between the scalar cosets based on the non-compact and the maximally non-compact numerator groups is the parametrization one can choose for the coset representatives. For the general non-compact real forms one can make use of the solvable Lie algebra gauge 
[17] to parameterize the scalar coset. The solvable Lie algebra is a subalgebra of the Borel algebra of $g$ (the Lie algebra of $G$ ). It is simply composed of certain Cartan and positive root generators of $g$ [18].

The bosonic lagrangian of the $D=10, \mathcal{N}=1$ abelian Yang-Mills supergravity which is coupled to $N U(1)$ gauge multiplets is $[8,9,22]$

$$
\mathcal{L}_{10}=R * 1-\frac{1}{2} * d \phi_{1} \wedge d \phi_{1}-\frac{1}{2} e^{\phi_{1}} * F_{(3)} \wedge F_{(3)}-\frac{1}{2} e^{\frac{1}{2} \phi_{1}} \sum_{I=1}^{N} * G_{(2)}^{I} \wedge G_{(2)}^{I},
$$

where $G_{(2)}^{I}=d B_{(1)}^{I}$ are the $N U(1)$ gauge field strengths. In (2.1) $\phi_{1}$ is a scalar field and the subscripts for the rest of the fields and the field strengths denote the degree of the fields. We also define the field strength of the field $A_{(2)}$ as

$$
F_{(3)}=d A_{(2)}+\frac{1}{2} B_{(1)}^{I} \wedge d B_{(1)}^{I} .
$$

The pure supergravity sector of (2.1) can be truncated from the IIA supergravity [2, 3, 4] by choosing the extra $R-R$ fields to be zero in the IIA lagrangian. The Kaluza-Klein ansatz for the ten dimensional spacetime metric upon $T^{n}$-reduction where $n=10-D$ is [24]

$$
d s_{10}^{2}=e^{\vec{s} \cdot \vec{\phi}^{\prime}} d s_{D}^{2}+\sum_{i=2}^{11-D} e^{2 \vec{\gamma}_{i} \cdot \vec{\phi}^{\prime}}\left(h^{i}\right)^{2}
$$

Here we have

$$
\begin{aligned}
\vec{s} & =\left(s_{2}, s_{3}, \ldots, s_{(11-D)}\right), \\
\vec{f}_{i} & =\left(0, \ldots, 0,(10-i) s_{i}, s_{i+1}, \ldots, s_{(11-D)}\right),
\end{aligned}
$$


where in the second line there are $i-2$ zeros and

$$
s_{i}=\sqrt{\frac{2}{((10-i)(9-i))}} .
$$

Also we define

$$
\vec{\gamma}_{i}=\frac{1}{2} \vec{s}-\frac{1}{2} \vec{f}_{i}
$$

and $h^{i}$ is

$$
h^{i}=d z^{i}+\mathcal{A}_{(1)}^{i}+\mathcal{A}_{(0) j}^{i} d z^{j},
$$

where $\mathcal{A}_{(1)}^{i}$ are the $D$-dimensional Maxwell gauge fields and $\mathcal{A}_{(0) j}^{i}$ are the $D$ dimensional scalars. The coordinates $\left\{z^{i}\right\}$ are the coordinates on the $n$-torus $T^{n}$. We further define the vector

$$
\overrightarrow{\phi^{\prime}}=\left(\phi_{2}, \phi_{3}, \ldots, \phi_{(11-D)}\right)
$$

whose components are the dilatons of the Kaluza-Klein reduction.

We should also mention how to built up an ansatz to reduce a general $D$-dimensional $(n-1)$-form potential field $A_{(n-1)}^{D}$ on $S^{1}$, with the assumption that the $D$-dimensional spacetime is composed of the Cartesian product of a $(D-1)$-dimensional subspacetime and $S^{1}$. The ansatz can be chosen as 21$]$

$$
A_{(n-1)}^{D}(x, z)=A_{(n-1)}(x)+A_{(n-2)}(x) \wedge d z,
$$

where the coordinates $x$ are on the $(D-1)$-dimensional spacetime and $z$ is the coordinate on $S^{1}$. When we take the exterior derivative of (2.9) we get

$$
\begin{aligned}
F_{(n)}^{D}(x, z) & =d A_{(n-1)}^{D}(x, z) \\
& =d A_{(n-1)}(x)+d A_{(n-2)}(x) \wedge d z .
\end{aligned}
$$

While choosing the $(D-1)$-dimensional field strength of $A_{(n-2)}$ as $F_{(n-1)}=$ 
$d A_{(n-2)}$, one does not simply choose the $(D-1)$-dimensional field strength of $A_{(n-1)}$ as $F_{(n)}=d A_{(n-1)}$ but for the purpose of obtaining a nice looking lower dimensional lagrangian one defines the lower dimensional field strengths through the transgression relations

$$
\begin{aligned}
F_{(n)}(x) & =d A_{(n-1)}(x)-d A_{(n-2)}(x) \wedge \mathcal{A}(x), \\
F_{(n-1)}(x) & =d A_{(n-2)}(x) .
\end{aligned}
$$

In terms of these $(D-1)$-dimensional field strengths the $D$-dimensional field strength can be given as

$$
F_{(n)}^{D}(x, z)=F_{(n)}(x)+F_{(n-1)}(x) \wedge(d z+\mathcal{A}(x)) .
$$

We can express the $D$-dimensional kinetic term of $F_{(n)}^{D}$ in terms of the $(D-1)$ dimensional field strengths we have defined as

$$
\begin{aligned}
\mathcal{L}_{F}^{D}= & -\frac{1}{2} * F_{(n)}^{D} \wedge F_{(n)}^{D} \\
= & \left(-\frac{1}{2} e^{-2(n-1) \alpha \phi} *^{(D-1)} F_{(n)} \wedge F_{(n)}\right. \\
& \left.-\frac{1}{2} e^{2(D-n-1) \alpha \phi} *^{(D-1)} F_{(n-1)} \wedge F_{(n-1)}\right) \wedge d z \\
= & \mathcal{L}_{F}^{(D-1)} \wedge d z .
\end{aligned}
$$

If we perform the $S^{1}$-reduction step by step on the ten dimensional metric by using the ansatz (2.3) and on the other fields in (2.1) as well as on the by-product lower dimensional fields by using the ansatz (2.9) then the ten 
dimensional lagrangian (2.1) can be written as

$$
\mathcal{L}_{10}=\mathcal{L}_{D} \wedge d z^{1} \wedge \cdots \wedge d z^{n}
$$

where the $S^{1}$-reduction is performed $n$ times. The $D$-dimensional lagrangian in (2.14) can be calculated in terms of the $D$-dimensional fields as

$$
\begin{aligned}
\mathcal{L}_{D}= & R * 1-\frac{1}{2} * d \vec{\phi} \wedge d \vec{\phi}-\frac{1}{2} e^{\vec{a}_{1} \cdot \vec{\phi}} * F_{(3)} \wedge F_{(3)} \\
& -\frac{1}{2} \sum_{i} e^{\vec{a}_{1 i} \cdot \vec{\phi}} * F_{(2) i} \wedge F_{(2) i}-\frac{1}{2} \sum_{i<j} e^{\vec{a}_{1 i j} \cdot \vec{\phi}} * F_{(1) i j} \wedge F_{(1) i j} \\
& -\frac{1}{2} \sum_{I} e^{\vec{c} \cdot \vec{\phi}} * G_{(2)}^{I} \wedge G_{(2)}^{I}-\frac{1}{2} \sum_{i, I} e^{\vec{c}_{i} \cdot \vec{\phi}} * G_{(1) i}^{I} \wedge G_{(1) i}^{I} \\
& -\frac{1}{2} \sum_{i} e^{\vec{b}_{i} \cdot \vec{\phi}} * \mathcal{F}_{(2)}^{i} \wedge \mathcal{F}_{(2)}^{i}-\frac{1}{2} \sum_{i<j} e^{\vec{b}_{i j} \cdot \vec{\phi}} * \mathcal{F}_{(1) j}^{i} \wedge \mathcal{F}_{(1) j}^{i},
\end{aligned}
$$

where $i, j=2, \ldots, 11-D$ and $\vec{\phi}=\left(\phi_{1}, \phi_{2}, \ldots, \phi_{(11-D)}\right)$ in which except $\phi_{1}$, the rest of the scalars are the Kaluza-Klein scalars which originate from the ten dimensional spacetime metric. The transgression relations which define the field strengths in (2.15) in terms of the potentials are as follows

$$
\begin{aligned}
F_{(3)}= & d A_{(2)}+\frac{1}{2} B_{(1)}^{I} d B_{(1)}^{I}-\left(d A_{(1) i}+\frac{1}{2} B_{(0) i}^{I} d B_{(1)}^{I}+\frac{1}{2} B_{(1)}^{I} d B_{(0) i}^{I}\right) \gamma_{j}^{i} \mathcal{A}_{(1)}^{j} \\
& +\frac{1}{2}\left(d A_{(0) i j}-B_{(0)[i}^{I} d B_{(0) j]}^{I}\right) \gamma_{k}^{i} \mathcal{A}_{(1)}^{k} \gamma_{m}^{j} \mathcal{A}_{(1)}^{m} \\
F_{(2) i}= & \gamma_{i}^{k}\left(d A_{(1) k}+\frac{1}{2} B_{(0) k}^{I} d B_{(1)}^{I}+\frac{1}{2} B_{(1)}^{I} d B_{(0) k}^{I}\right.
\end{aligned}
$$




$$
\begin{gathered}
\left.+\left(d A_{(0) k j}-B_{(0)[k}^{I} d B_{(0) j]}^{I}\right) \gamma_{l}^{j} \mathcal{A}_{(1)}^{l}\right), \\
F_{(1) i j}=\gamma_{i}^{l} \gamma_{j}^{m}\left(d A_{(0) l m}-B_{(0)[l}^{I} d B_{(0) m]}^{I}\right), \quad \mathcal{F}_{(2)}^{i}=\widetilde{\gamma}_{j}^{i} d\left(\gamma_{k}^{j} \mathcal{A}_{(1)}^{k}\right), \\
G_{(2)}^{I}=d B_{(1)}^{I}-d B_{(0) i}^{I} \gamma_{j}^{i} \mathcal{A}_{(1)}^{j}, \quad G_{(1) i}^{I}=\gamma_{i}^{j} d B_{(0) j}^{I}, \quad \mathcal{F}_{(1) j}^{i}=\gamma_{j}^{k} d \mathcal{A}_{(0) k}^{i},
\end{gathered}
$$

where we have omitted the wedge product. The dilatons $\overrightarrow{\phi^{\prime}}$, the Kaluza-KleinMaxwell potentials $\mathcal{A}_{(1)}^{j}$ as well as the axions $\mathcal{A}_{(0) k}^{i}$ in each dimension are the descendants of the ten dimensional spacetime metric. The potentials $A_{(0) l m}$, $A_{(1) k}$ and $A_{(2)}$ are the Kaluza-Klein descendants of the two-form potential in $D=10$. The potentials $B_{(0) j}^{I}$ and $B_{(1)}^{I}$ are the $D$-dimensional remainders of the ten dimensional Yang-Mills potentials $B_{(1)}^{I}$ as a result of the ansatz (2.9) applied in each $S^{1}$-reduction step. We define the matrix $\gamma_{j}^{i}$ as

$$
\gamma_{j}^{i}=\left[\left(1+\mathcal{A}_{(0)}\right)^{-1}\right]_{j}^{i}
$$

and $\widetilde{\gamma}_{j}^{i}$ is the inverse of it. The dilaton vectors which couple to the scalars $\vec{\phi}$ in various field strength terms in (2.15) are defined as [22]

$$
\begin{gathered}
\vec{a}_{1}=(1,-2 \vec{s}) \quad, \quad \vec{a}_{1 i}=\left(1, \vec{f}_{i}-2 \vec{s}\right) \quad, \quad \vec{a}_{1 i j}=\left(1, \vec{f}_{i}+\vec{f}_{j}-2 \vec{s}\right) \\
\vec{b}_{i}=\left(0,-\vec{f}_{i}\right) \quad, \quad \vec{b}_{i j}=\left(0,-\vec{f}_{i}+\vec{f}_{j}\right) \\
\vec{c}=\left(\frac{1}{2},-\vec{s}\right) \quad, \quad \vec{c}_{i}=\left(\frac{1}{2}, \vec{f}_{i}-\vec{s}\right) .
\end{gathered}
$$

In [22] the scalar sector and the abelian matter coupling in each dimension are reformulated through a series of field and field strength redefinitions. The dualisation of certain fields is also performed in various dimensions when 
necessary. In this way the global symmetry groups are identified. Also the lagrangian (2.15) is written in a more compact form whose scalar sector is formulated as a $G / K$ non-linear sigma model. We will not mention about the details of this calculation here, we will only present the result which will be a reference point for our further analysis in the following sections. In [22] it is shown that when a single dilaton is decoupled from the rest of the scalars then the $G / K$ coset representatives $\nu^{\prime}$ and the internal metric

$$
\mathcal{M}=\nu^{\prime T} \nu^{\prime}
$$

generated by the remaining scalars are elements of $O^{\prime}(10-D+N, 10-D) 1$ which is composed of the $(20-2 D+N)$-dimensional real matrices $A$ which satisfy

$$
A^{T} \Omega A=\Omega,
$$

where the $(20-2 D+N) \times(20-2 D+N)$ matrix $\Omega$ is

$$
\Omega=\left(\begin{array}{ccc}
0 & 0 & -\mathbf{1}_{(10-D)} \\
0 & \mathbf{1}_{(N)} & 0 \\
-\mathbf{1}_{(10-D)} & 0 & 0
\end{array}\right),
$$

in which $\mathbf{1}_{(n)}$ is the $n \times n$ unit matrix. The scalar lagrangian of the $D$ dimensional compactified theories can be described in the form

$$
\mathcal{L}_{\text {scalar }}=\frac{1}{4} \operatorname{tr}\left(* d \mathcal{M}^{-1} \wedge d \mathcal{M}\right),
$$

with an additional decoupled dilatonic kinetic term after certain field redefinitions. The coset representatives $\nu^{\prime}$ are parameterized as

$$
\nu^{\prime}=e^{\frac{1}{2} \varphi^{i} H_{i}} e^{A_{(0) j}^{i} E_{i}^{j}} e^{\frac{1}{2} A_{(0) i j} V^{i j}} e^{B_{(0) i}^{I} U_{I}^{i}} .
$$

\footnotetext{
${ }^{1}$ The reason why we use a prime will be clear later. Here we slightly change the notation used in 22 .
} 
Here $\left\{H_{i}, E_{i}^{j}, V^{i j}, U_{I}^{i}\right\}$ are $(20-2 D+N)$-dimensional matrices and following the notation of [22] we have modified the ranges of the indices $i, j$ as $i, j=$ $1, \ldots, 10-D$. We also assume that $i<j$. The matrices $\left\{H_{i}, E_{i}^{j}, V^{i j}, U_{I}^{i}\right\}$ are [22, 25]

$$
\begin{gathered}
\vec{H}_{i}=\left(\begin{array}{ccc}
\sum_{i} \vec{c}_{i} e_{i i} & 0 & 0 \\
0 & 0 & 0 \\
0 & 0 & -\sum_{i} \vec{c}_{i} e_{i i}
\end{array}\right), \quad E_{i}^{j}=\left(\begin{array}{ccc}
-e_{j i} & 0 & 0 \\
0 & 0 & 0 \\
0 & 0 & e_{i j}
\end{array}\right), \\
V^{i j}=\left(\begin{array}{ccc}
0 & 0 & e_{i j}-e_{j i} \\
0 & 0 & 0 \\
0 & 0 & 0
\end{array}\right), \quad U_{I}^{i}=\left(\begin{array}{ccc}
0 & e_{i I} & 0 \\
0 & 0 & e_{I i} \\
0 & 0 & 0
\end{array}\right),
\end{gathered}
$$

where the matrices are partitioned with appropriate dimensions which can be read from the non-zero entries and $e_{a b}$ is a matrix with appropriate dimensions and which has zero entries, except a $\{+1\}$ entry at the $a^{\prime}$ th row and the $b$ 'th column. Notice that the indices $i, j=1, \ldots, 10-D$ and $I=1, \ldots, N$ determine the dimensions of the matrices $e_{a b}$. As it is mentioned in [22] the matrices $\left\{H_{i}, E_{i}^{j}, V^{i j}, U_{I}^{i}\right\}$ form up an algebra and they can be embedded in a fundamental representation of $o^{\prime}(10-D+N, 10-D)$. As a matter of fact they generate the solvable Lie algebra of $o^{\prime}(10-D+N, 10-D)$ in each dimension thus the parametrization of the coset in (2.23) is nothing but an example of the solvable Lie algebra gauge. This result justifies the prediction that the global internal symmetry group of the scalar lagrangian is $O^{\prime}(10-D+N, 10-D)$ and the scalar manifold for the $D$-dimensional compactified theory with $N$ gauge multiplet couplings becomes

$$
\frac{O^{\prime}(10-D+N, 10-D)}{O(10-D+N) \times O(10-D)} \times \mathbb{R}
$$

The extra $\mathbb{R}$ factor arises since there is an additional dilaton which is decoupled from the rest of the scalars in the scalar lagrangian. The group 
$O^{\prime}(10-D+N, 10-D) \times \mathbb{R}$ is the global internal symmetry of not only the scalar lagrangian but the entire $D$-dimensional bosonic lagrangian as well. The lagrangian (2.15) upon the above mentioned restoration, in terms of the newly defined fields can be written as

$$
\begin{aligned}
\mathcal{L}_{D}= & R * 1-\frac{1}{2} * d \phi \wedge d \phi+\frac{1}{4} \operatorname{tr}\left(* d \mathcal{M}^{-1} \wedge d \mathcal{M}\right) \\
& -\frac{1}{2} e^{-\sqrt{8 /(D-2)} \phi} * F_{(3)} \wedge F_{(3)}-\frac{1}{2} e^{-\sqrt{2 /(D-2)} \phi} * H_{(2)}^{T} \wedge \mathcal{M} H_{(2)},
\end{aligned}
$$

where

$$
H_{(2)}=d C_{(1)},
$$

in which we define

$$
C_{(1)}=\left(\begin{array}{c}
A_{(1) i} \\
B_{(1)}^{I} \\
\mathcal{A}_{(1)}^{i}
\end{array}\right),
$$

which is a column vector of dimension $(20-2 D+N)$. The field strength $F_{(3)}$ in (2.26) can be given in a compact form for any dimension as

$$
F_{(3)}=d A_{(2)}+\frac{1}{2} C_{(1)}^{T} \Omega d C_{(1)} .
$$

The definitions of the potentials introduced in (2.26) in terms of the original potentials which come from the Kaluza-Klein reduction in (2.15) can be referred in [22]. In this work, to derive the field equations in the following sections we will consider the potentials used to construct (2.26) as our starting point. In addition to the general $D$-dimensional lagrangian (2.26) furthermore the $D=4$ and the $D=3$ cases can be studied separately, since they have global symmetry enhancements over the general scheme of $O^{\prime}(10-D+N, 10-D) \times \mathbb{R}[22$. One may define additional scalars in these dimensions by dualizing certain fields by applying the lagrange multiplier 
methods. When the two-form potential $A_{(2)}$ is dualized with an additional axion in $D=4$, an axion-dilaton, $S L(2, \mathbb{R})$ system is decoupled from the rest of the scalars in the scalar lagrangian and the enlarged $D=4$ scalar manifold becomes

$$
\frac{O^{\prime}(6+N, 6)}{O(6) \times O(6+N)} \times \frac{S L(2, \mathbb{R})}{O(2)} .
$$

On the other hand in $D=3$, the bosonic fields $\left(\mathcal{A}_{(1)}^{i}, A_{(1) i}, B_{(1)}^{I}\right)$ can be dualized to give $7+7+N$ additional axions. In this case the entire bosonic sector comes out to be composed of only the scalars. The $D=3$ enlarged scalar manifold then becomes

$$
\frac{O^{\prime}(8+N, 8)}{O(8) \times O(8+N)}
$$

We see that all of the global internal symmetry groups in (2.25), (2.30) and (2.31) apart from the contributions of the decoupled scalars namely the groups $O^{\prime}(10-D+N, 10-D), O^{\prime}(6+N, 6), O^{\prime}(8+N, 8)$ are non-compact real forms of semi-simple Lie groups and they enable solvable Lie algebra parametrizations of the cosets that are generated by the denominator groups $O(10-D+N) \times O(10-D), O(6) \times O(6+N), O(8) \times O(8+N)$, respectively.

\section{The General Formalism for the SSSM with Couplings}

In this section we will construct the general formulation of the abelian gauge matter, dilaton and Chern-Simons couplings of the generic symmetric space sigma model (SSSM) [19, 26, 27, 28, 29] under the solvable Lie algebra parametrization. We will derive the field equations for a general theory and then we will give a number of examples on which we can apply the results. Also in the next section we will use the results to write down the field equa- 
tions of the $D$-dimensional low energy effective theory of the $E_{8} \times E_{8}$ heterotic string which is studied in the previous section. The general formalism and the field equations are already derived for the symmetric space sigma model and the abelian matter coupled symmetric space sigma model in [28, 29] and [23. respectively under the solvable Lie algebra gauge. Therefore in this section we extend the construction of [23] further by including a dilaton and a Chern-Simons coupling.

We will first construct the scalar lagrangian by using the solvable Lie algebra parametrization [17] for the coset representatives. In the last section we have seen that the toroidally compactified heterotic string gives the MaxwellEinstein supergravities in $D$-dimensions. The scalars decoupled from a single dilaton are governed by symmetric space sigma models. The scalar fields parameterize the scalar coset manifold $O^{\prime}(10-D+N, 10-D) / O(10-D+$ $N) \times O(10-D)$ where $O^{\prime}(10-D+N, 10-D)$ is in general a non-compact real form of a semi-simple Lie group and $O(10-D+N) \times O(10-D)$ is its maximal compact subgroup. For this reason the scalar manifold is a Riemannian globally symmetric space for all the $O^{\prime}(10-D+N, 10-D)$-invariant Riemannian structures on it [18].

In this section we will use a slightly different notation. We will take the representation of the global symmetry group as $O(10-D, 10-D+N)$ whose $(20-2 D+N)$-dimensional real matrix elements $A$ satisfy the defining relation

$$
A^{T} \eta A=\eta
$$

where

$$
\eta=\operatorname{diag}(-,-, \ldots,-,+,+, \ldots,+),
$$

is the indefinite signature metric with $10-D$ minus signs and $10-D+N$ plus signs. Although the set of matrices defined in (2.20) and (3.1) may differ the groups they form are isomorphic to each other [30]. Thus in this section we will base our formulation on the scalar coset which is the conventional one 
used in the construction of the Maxwell-Einstein supergravities in [31, 32, 33].

To construct the symmetric space sigma model lagrangian for the scalar coset manifold,

$$
\frac{O(10-D, 10-D+N)}{O(10-D) \times O(10-D+N)},
$$

one may make use of the solvable Lie algebra parametrization [17] for the parametrization of the coset representatives. The solvable Lie algebra gauge or the parametrization is a consequence of the Iwasawa decomposition [18]

$$
\begin{aligned}
o(10-D, 10-D+N) & =\mathbf{k}_{0} \oplus \mathbf{s}_{0} \\
& =\mathbf{k}_{0} \oplus \mathbf{h}_{\mathbf{k}} \oplus \mathbf{n}_{\mathbf{k}},
\end{aligned}
$$

where $\mathbf{k}_{0}$ is the Lie algebra of $O(10-D) \times O(10-D+N)$ which is a maximal compact subgroup of $O(10-D, 10-D+N)$ and $\mathbf{s}_{0}$ is a solvable Lie subalgebra of $o(10-D, 10-D+N)$. In (3.4) $\mathbf{h}_{k}$ is a subalgebra of the Cartan subalgebra $\mathbf{h}_{0}$ of $o(10-D, 10-D+N)$ which generates the maximal R-split torus in $O(10-D, 10-D+N)$ [18, 27, 29]. The nilpotent Lie subalgebra $\mathbf{n}_{\mathbf{k}}$ of $o(10-D, 10-D+N)$ is generated by a subset $\left\{E_{m}\right\}$ of the positive root generators of $o(10-D, 10-D+N)$ where $m \in \Delta_{n c}^{+}$. The roots in $\Delta_{n c}^{+}$are the non-compact roots with respect to the Cartan involution $\theta$ induced by the Cartan decomposition [18, 23, 29]

$$
o(10-D, 10-D+N)=\mathbf{k}_{0} \oplus \mathbf{u}_{0},
$$

where $\mathbf{u}_{0}$ is a vector subspace of $o(10-D, 10-D+N)$. We should remark that when the Lie group $O(10-D, 10-D+N)$ is in split real form (maximally non-compact) [18] then for a Cartan decomposition and a resulting Iwasawa decomposition the solvable Lie algebra $\mathbf{s}_{0}$ in (3.4) coincides with the Borel subalgebra which is generated by all the simple root Cartan generators $\left\{H_{\alpha}\right\}$ (or any other basis) of $\mathbf{h}_{\mathbf{0}}$ and the positive root generators $\left\{E_{\alpha} \mid \alpha \in \Delta^{+}\right\}$ 
[19]. The split real form global symmetry groups are of the form $O(n, n)$, $O(n+1, n), O(n, n+1)$ [21].

Since we have [21]

$$
\operatorname{dims}_{0}=(10-D) \times(10-D+N),
$$

by coupling $(10-D) \times(10-D+N)$ scalars to the generators of the solvable Lie algebra $\mathbf{s}_{0}$ we can parameterize the representatives of the scalar coset manifold in the solvable Lie algebra gauge as [18]

$$
\nu=e^{\frac{1}{2} \phi^{i} H_{i}} e^{\chi^{m} E_{m}},
$$

where $\left\{H_{i}\right\}$ for $i=1, \ldots, \operatorname{dim}\left(\mathbf{h}_{k}\right) \equiv r$ are the generators of $\mathbf{h}_{k}$ and $\left\{E_{m}\right\}$ for $m \in \Delta_{n c}^{+}$are the positive root generators which generate $\mathbf{n}_{\mathbf{k}}$. The scalars $\left\{\phi^{i}\right\}$ for $i=1, \ldots, r$ are called the dilatons and $\left\{\chi^{m}\right\}$ for $m \in \Delta_{n c}^{+}$are called the axions. The coset representatives $\nu$ satisfy the defining relation of $O(10-$ $D, 10-D+N)$ namely

$$
\nu^{T} \eta \nu=\eta .
$$

If we assume that we choose the $(20-2 D+N)$-dimensional fundamental representation for the algebra $o(10-D, 10-D+N)$ and if we define the internal metric

$$
\mathcal{M}=\nu^{T} \nu,
$$

then the scalar lagrangian which governs the $(10-D) \times(10-D+N)$ scalar fields of the theory can be constructed as

$$
\mathcal{L}_{\text {scalar }}=\frac{1}{4} \operatorname{tr}\left(* d \mathcal{M}^{-1} \wedge d \mathcal{M}\right) .
$$

Since in this work our main perspective is the derivation of the field equations of the toroidally compactified heterotic string we take the scalar coset as $O(10-D, 10-D+N) / O(10-D) \times O(10-D+N)$ however it is obvious 
that our analysis is valid for any scalar coset of the form $O(p, q) / O(p) \times O(q)$. As we have assumed a $(20-2 D+N)$-dimensional representation for the Lie algebra $o(10-D, 10-D+N)$ we can couple $(20-2 D+N)$ abelian gauge fields $A^{I}$ and a dilaton $\sigma$ to the scalars as [23, 26, 27, 28, 29]

$$
\begin{aligned}
\mathcal{L}_{m} & =c_{2} e^{\alpha_{1} \sigma} * F \wedge \mathcal{M} F \\
& =c_{2} e^{\alpha_{1} \sigma} \mathcal{M}_{I J} * F^{I} \wedge F^{J},
\end{aligned}
$$

where the field strengths of the gauge fields are defined to be $F^{I}=d A^{I}$. Furthermore one can couple a two-form field $B$ to the other field content by introducing the Chern-Simons three-form

$$
G=d B+c_{4} \eta_{I J} A^{I} \wedge F^{J}
$$

which becomes the field strength of $B$ indeed. We are ready to write down the general lagrangian of the $O(p, q) / O(p) \times O(q)$ scalar coset which has the abelian gauge, Chern-Simons and the dilaton couplings in $D$-dimensional spacetime,

$$
\begin{aligned}
\mathcal{L}= & c_{1} * d \sigma \wedge d \sigma+c_{2} e^{\alpha_{1} \sigma} * F \wedge \mathcal{M} F \\
& +\frac{1}{4} \operatorname{tr}\left(* d \mathcal{M}^{-1} \wedge d \mathcal{M}\right)+c_{3} e^{\alpha_{2} \sigma} * G \wedge G .
\end{aligned}
$$

Our field content becomes $\left\{\sigma, A^{I}, B, \phi^{j}, \chi^{m}\right\}$ in which we have $(10-D) \times$ $(10-D+N)$ scalars of the coset, $(20-2 D+N)$ abelian gauge fields $A^{I}$, a two-form $B$ and a single dilaton $\sigma$ which we take to be decoupled from the rest of the scalars that parameterize the scalar coset manifold.

We will first vary the lagrangian (3.13) with respect to the dilaton $\sigma$. The Euler-Lagrange equation [34] which yields the vanishing of the variation of 
(3.13) with respect to $\sigma$ gives

$$
\begin{aligned}
(-1)^{(D-1)} d\left(2 c_{1} * d \sigma\right)= & c_{3} \alpha_{2} e^{\alpha_{2} \sigma} * G \wedge G \\
& +c_{2} \alpha_{1} e^{\alpha_{1} \sigma} \mathcal{M}_{I J} * F^{I} \wedge F^{J}
\end{aligned}
$$

The left hand side comes from the variation of the kinetic term of the dilaton in (3.13) and the terms in the right hand side arise due to the coupling of the dilaton to the other fields. Since the lagrangian (3.13) does not explicitly depend on $B$ the variation of it with respect to $B$ will yield the Euler-Lagrange equation which defines a closed form. It is

$$
-(-1)^{(D+1)} d\left(c_{3} e^{\alpha_{2} \sigma}\left(2 * d B+2 c_{4} \eta_{I J} *\left(A^{I} \wedge F^{J}\right)\right)\right)=0 .
$$

By using the definition of the Chern-Simons three-form given in (3.12) we can write $(3.15)$ as

$$
d\left(e^{\alpha_{2} \sigma} * G\right)=0 .
$$

We will now vary the lagrangian (3.13) with respect to the abelian gauge fields $A^{I}$. The Euler-Lagrange equations of motion read

$$
\begin{aligned}
0= & (-1)^{3(D-3)} 2 c_{3} c_{4} e^{\alpha_{2} \sigma} \eta_{I K} F^{K} \wedge\left(* d B+c_{4} \eta_{L M} *\left(A^{L} \wedge F^{M}\right)\right) \\
& +d\left(c_{2} e^{\alpha_{1} \sigma}\left(\mathcal{M}_{I J} * d A^{J}+\mathcal{M}_{J I} * d A^{J}\right)\right. \\
& \left.+2 c_{3} c_{4} e^{\alpha_{2} \sigma}\left(* d B+c_{4} \eta_{L M} *\left(A^{L} \wedge F^{M}\right)\right) \wedge \eta_{J I} A^{J}\right) .
\end{aligned}
$$

Bearing in mind that from its definition in (3.9) the internal metric $\mathcal{M}$ is symmetric so that

$$
\mathcal{M}_{I J}=\mathcal{M}_{J I}
$$


also by using the definition of the Chern-Simons form in (3.12) the EulerLagrange equations (3.17) for the gauge fields $A^{I}$ can be written as

$$
\begin{aligned}
(-1)^{3(D-3)} c_{3} c_{4} e^{\alpha_{2} \sigma} \eta_{I K} F^{K} \wedge * G= & -d\left(c_{2} e^{\alpha_{1} \sigma} \mathcal{M}_{I J} * d A^{J}\right) \\
& -d\left(c_{3} c_{4} e^{\alpha_{2} \sigma} * G \wedge \eta_{J I} A^{J}\right) .
\end{aligned}
$$

Now by acting the exterior derivative the last term in (3.19) can be written as

$$
\begin{aligned}
-d\left(c_{3} c_{4} e^{\alpha_{2} \sigma} * G \wedge \eta_{J I} A^{J}\right)= & -c_{3} c_{4}\left(d\left(e^{\alpha_{2} \sigma} * G\right) \wedge \eta_{J I} A^{J}\right. \\
& \left.+(-1)^{(D-3)} e^{\alpha_{2} \sigma} * G \wedge \eta_{J I} d A^{J}\right) .
\end{aligned}
$$

If we use the field equation (3.16) of the two-form field $B$ above and insert the result back in (3.19) we obtain

$$
\begin{aligned}
(-1)^{3(D-3)} c_{3} c_{4} e^{\alpha_{2} \sigma} \eta_{I K} F^{K} \wedge * G= & -d\left(c_{2} e^{\alpha_{1} \sigma} \mathcal{M}_{I J} * d A^{J}\right) \\
& -(-1)^{(D-3)} c_{3} c_{4} e^{\alpha_{2} \sigma} * G \wedge \eta_{J I} d A^{J} .
\end{aligned}
$$

Noting that $\eta$ is symmetric,

$$
\eta_{I J}=\eta_{J I}
$$

we can finally write the field equations of $A^{I}$ as

$$
d\left(c_{2} e^{\alpha_{1} \sigma} \mathcal{M}_{I J} * F^{J}\right)=(-1)^{D} 2 c_{3} c_{4} e^{\alpha_{2} \sigma} \eta_{I J} F^{J} \wedge * G
$$

which we will compactly express in matrix form as

$$
d\left(c_{2} e^{\alpha_{1} \sigma} \mathcal{M} * F\right)=(-1)^{D} 2 c_{3} c_{4} e^{\alpha_{2} \sigma} \eta F \wedge * G .
$$


One can take the internal metric out of the exterior derivative on the left hand side above to obtain

$$
\begin{aligned}
d\left(c_{2} e^{\alpha_{1} \sigma} * F\right)= & -c_{2} e^{\alpha_{1} \sigma} \mathcal{M}^{-1} d \mathcal{M} \wedge * F \\
& +(-1)^{D} 2 c_{3} c_{4} e^{\alpha_{2} \sigma} \mathcal{M}^{-1} \eta F \wedge * G
\end{aligned}
$$

We may also express the field equations (3.23) in terms of the Cartan-Maurer form

$$
\mathcal{G}=d \nu \nu^{-1} .
$$

Before going in that direction we should remark on certain identities which the general coset representatives (3.7) satisfy. First of all since

$$
\nu \nu^{-1}=\nu^{-1} \nu=\mathbf{1},
$$

taking the exterior derivative of both sides yields the identities

$$
d \nu \nu^{-1}=-\nu d \nu^{-1}, \quad d \nu^{-1} \nu=-\nu^{-1} d \nu .
$$

Also as $\eta$ is a symmetric matrix and

$$
\eta^{2}=1
$$

starting from (3.8) one can derive the following identities

$$
\begin{gathered}
\eta \nu^{T} \eta=\nu^{-1}, \quad \eta \nu^{-1} \eta=\nu^{T}, \quad \nu \eta \nu^{T}=\eta, \\
\nu^{-1} \eta\left(\nu^{T}\right)^{-1}=\eta, \quad \eta \nu \eta=\left(\nu^{T}\right)^{-1}, \quad \eta\left(\nu^{T}\right)^{-1} \eta=\nu .
\end{gathered}
$$

Since $\eta$ is a constant matrix one can take the exterior derivative of the above 
identities to obtain

$$
\eta d \nu^{T} \eta=d \nu^{-1}, \quad \eta d \nu^{-1} \eta=d \nu^{T}, \quad \eta d \nu \eta=d\left(\nu^{T}\right)^{-1}
$$

Due to the definition of the coset elements in (3.7) we also have

$$
\left(\nu^{T}\right)^{-1}=\left(\nu^{-1}\right)^{T}
$$

In order to write the field equations of $A^{I}$ in terms of the Cartan-Maurer form $\mathcal{G}$ starting from $(3.24)$ we can proceed as follows

$$
\begin{aligned}
d\left(c_{2} e^{\alpha_{1} \sigma} \mathcal{M} * F\right)= & c_{2} e^{\alpha_{1} \sigma} d \nu^{T} \wedge \nu * F \\
& +\nu^{T}\left(c_{2} e^{\alpha_{1} \sigma} d \nu \wedge * F+\nu d\left(c_{2} e^{\alpha_{1} \sigma} * F\right)\right) .
\end{aligned}
$$

Inserting this result back in (3.24) we obtain

$$
\begin{aligned}
d\left(c_{2} e^{\alpha_{1} \sigma} * F\right)= & -c_{2} e^{\alpha_{1} \sigma} \nu^{-1} d \nu \wedge * F \\
& -c_{2} e^{\alpha_{1} \sigma} \nu^{-1}\left(d \nu \nu^{-1}\right)^{T} \nu \wedge * F \\
& +(-1)^{D} 2 c_{3} c_{4} e^{\alpha_{2} \sigma} \nu^{-1}\left(\nu^{T}\right)^{-1} \eta F \wedge * G .
\end{aligned}
$$

Now multiplying the above equation with $\eta$ on both sides and then using the identities (3.28)-(3.32) effectively we obtain

$$
\begin{aligned}
d\left(c_{2} e^{\alpha_{1} \sigma} \eta * F\right)= & c_{2} e^{\alpha_{1} \sigma} d \nu^{T}\left(\nu^{T}\right)^{-1} \wedge \eta * F \\
& +c_{2} e^{\alpha_{1} \sigma} \nu^{T}\left(d \nu \nu^{-1}\right)\left(\nu^{T}\right)^{-1} \wedge \eta * F \\
& +(-1)^{D} 2 c_{3} c_{4} e^{\alpha_{2} \sigma} \mathcal{M} F \wedge * G
\end{aligned}
$$


By using

$$
d \nu^{T}\left(\nu^{T}\right)^{-1}=\nu^{T} \mathcal{G}^{T}\left(\nu^{T}\right)^{-1},
$$

finally we can express (3.24) in terms of the Cartan-Maurer form (3.26) as

$$
\begin{aligned}
d\left(c_{2} e^{\alpha_{1} \sigma} \eta * F\right)= & c_{2} e^{\alpha_{1} \sigma}\left(\nu^{T}\left(\mathcal{G}^{T}+\mathcal{G}\right)\left(\nu^{T}\right)^{-1}\right) \wedge \eta * F \\
& +(-1)^{D} 2 c_{3} c_{4} e^{\alpha_{2} \sigma} \mathcal{M} F \wedge * G
\end{aligned}
$$

For the generic non-split scalar cosets the Cartan-Maurer form $\mathcal{G}$ is calculated explicitly in terms of the coset scalars namely the dilatons $\left\{\phi^{i}\right\}$ and the axions $\left\{\chi^{m}\right\}$ in $[29]$. It reads

$$
\begin{aligned}
\mathcal{G} & =\frac{1}{2} d \phi^{i} H_{i}+e^{\frac{1}{2} \beta_{i} \phi^{i}} U^{\beta} E_{\beta} \\
& =\frac{1}{2} d \phi^{i} H_{i}+\overrightarrow{\mathbf{E}}^{\prime} \Omega \overrightarrow{d \chi}
\end{aligned}
$$

where $\left\{H_{i}\right\}$ for $i=1, \ldots, r$ are the generators of $\mathbf{h}_{k}$ and $\left\{E_{\beta}\right\}$ for $\beta \in \Delta_{n c}^{+}$are the generators of $\mathbf{n}_{k}$ as we have already defined before. We have introduced the column vector

$$
U^{\alpha}=\Omega_{\beta}^{\alpha} d \chi^{\beta}
$$

and the row vector

$$
\left(\overrightarrow{\mathbf{E}^{\prime}}\right)_{\beta}=e^{\frac{1}{2} \beta_{i} \phi^{i}} E_{\beta},
$$

where the components of the roots $\beta \in \Delta_{n c}^{+}$are defined as

$$
\left[H_{i}, E_{\beta}\right]=\beta_{i} E_{\beta}
$$


Also $\Omega$ is a $\operatorname{dim} \mathbf{n}_{\mathbf{k}} \times \operatorname{dim} \mathbf{n}_{\mathbf{k}}$ matrix

$$
\begin{aligned}
\boldsymbol{\Omega} & =\sum_{m=0}^{\infty} \frac{\omega^{m}}{(m+1) !} \\
& =\left(e^{\omega}-I\right) \omega^{-1} .
\end{aligned}
$$

The components of the matrix $\omega$ are

$$
\omega_{\beta}^{\gamma}=\chi^{\alpha} K_{\alpha \beta}^{\gamma}
$$

The structure constants $K_{\alpha \beta}^{\gamma}$ are defined as

$$
\left[E_{\alpha}, E_{\beta}\right]=K_{\alpha \beta}^{\gamma} E_{\gamma}
$$

In other words since

$$
\left[E_{\alpha}, E_{\beta}\right]=N_{\alpha, \beta} E_{\alpha+\beta}
$$

we have

$$
\begin{gathered}
K_{\beta \gamma}^{\alpha}=N_{\beta, \gamma} \text { if } \beta+\gamma=\alpha \quad, \quad K_{\beta \gamma}^{\alpha}=0 \quad \text { if } \beta+\gamma \neq \alpha, \\
K_{\beta \beta}^{\alpha}=0 .
\end{gathered}
$$

The reason why we have written the field equations of the gauge fields $A^{I}$ (3.24) in terms of the Cartan-Maurer form $\mathcal{G}$ in (3.37) becomes clear now. After choosing a $(20-2 D+N)$-dimensional representation for the Lie algebra $o(10-D, 10-D+N)$ (thus for the solvable Lie subalgebra $\mathbf{s}_{0}$ ) and after expressing the coset representatives (3.7) and the internal metric (3.9) as matrices one can insert (3.38) in (3.37) to write the later explicitly in terms of the coset scalars $\phi^{i}$ and $\chi^{m}$.

The terms in the lagrangian (3.13) which contain the coset scalars $\phi^{i}$ and 
$\chi^{m}$ are

$$
\mathcal{L}\left(\phi^{i}, \chi^{m}\right)=\frac{1}{4} \operatorname{tr}\left(* d \mathcal{M}^{-1} \wedge d \mathcal{M}\right)+c_{2} e^{\alpha_{1} \sigma} * F \wedge \mathcal{M} F
$$

By using the results of [26, 28, 29] we can write (3.47) as

$$
\mathcal{L}\left(\phi^{i}, \chi^{m}\right)=-\frac{1}{2} \operatorname{tr}\left(* \mathcal{G} \wedge \mathcal{G}^{T}+* \mathcal{G} \wedge \mathcal{G}\right)+c_{2} e^{\alpha_{1} \sigma} * F \wedge \mathcal{M} F
$$

Furthermore by using (3.38) we have

$$
\begin{aligned}
\mathcal{L}\left(\phi^{i}, \chi^{m}\right)= & -\frac{1}{2} \sum_{i=1}^{r} * d \phi^{i} \wedge d \phi^{i}-\frac{1}{2} \sum_{\beta \in \Delta_{n c}^{+}} e^{\beta_{i} \phi^{i}} * U^{\beta} \wedge U^{\beta} \\
& +c_{2} e^{\alpha_{1} \sigma} * F \wedge \mathcal{M} F
\end{aligned}
$$

Following the outline of [26, 27] which treat $U^{\alpha}$ as independent fields and then use the Lagrange multiplier formalism the field equations of $\phi^{i}$ and $\chi^{m}$ arising from the variation of (3.49) have been derived in [23]. They can be given as

$$
\begin{aligned}
d\left(e^{\frac{1}{2} \gamma_{i} \phi^{i}} * U^{\gamma}\right)= & -\frac{1}{2} \gamma_{j} e^{\frac{1}{2} \gamma_{i} \phi^{i}} d \phi^{j} \wedge * U^{\gamma} \\
& +\sum_{\tau-\beta=-\gamma} e^{\frac{1}{2} \tau_{i} \phi^{i}} e^{\frac{1}{2} \beta_{i} \phi^{i}} N_{\tau,-\beta} U^{\tau} \wedge * U^{\beta} \\
d\left(* d \phi^{i}\right)= & \frac{1}{2} \sum_{\beta \in \Delta_{n c}^{+}} \beta_{i} e^{\frac{1}{2} \beta_{j} \phi^{j}} U^{\beta} \wedge e^{\frac{1}{2} \beta_{j} \phi^{j}} * U^{\beta} \\
& +(-1)^{D} c_{2} e^{\alpha_{1} \sigma}\left(\left(H_{i}\right)_{N}^{A} \nu_{M}^{N} \nu_{J}^{A}\right) * F^{M} \wedge F^{J}
\end{aligned}
$$

$\tau, \beta, \gamma \in \Delta_{n c}^{+}$and $i=1, \ldots, \operatorname{dim}\left(\mathbf{h}_{k}\right) \equiv r$. The matrices $\left\{\left(H_{i}\right)_{N}^{A}\right\}$ are the 
ones corresponding to the generators $\left\{H_{i}\right\}$ in the $(20-2 D+N)$-dimensional representation chosen. By also referring to [28] the field equations in (3.50) can more compactly be written as

$$
\begin{aligned}
d\left(e^{\gamma_{i} \phi^{i}} * U^{\gamma}\right)= & \sum_{\alpha-\beta=-\gamma} N_{\alpha,-\beta} U^{\alpha} \wedge e^{\beta_{i} \phi^{i}} * U^{\beta}, \\
d\left(* d \phi^{i}\right)= & \frac{1}{2} \sum_{\beta \in \Delta_{n c}^{+}} \beta_{i} e^{\frac{1}{2} \beta_{j} \phi^{j}} U^{\beta} \wedge e^{\frac{1}{2} \beta_{j} \phi^{j}} * U^{\beta} \\
& +(-1)^{D} c_{2} e^{\alpha_{1} \sigma} * F \wedge \nu^{T} H_{i} \nu F .
\end{aligned}
$$

It is worth stating that the last term in the second equation above arises from the variation of the matter lagrangian (3.11) with respect to the dilaton $\phi^{i}$. A similar term is missing for the axions $\chi^{m}$ owing to the Lagrange multiplier method used in the derivation of the corresponding field equations [26, 28]. Therefore we have

$$
(-1)^{D} c_{2} e^{\alpha_{1} \sigma} * F \wedge \nu^{T} H_{i} \nu F=(-1)^{D} \frac{\partial \mathcal{L}_{m}}{\partial \phi^{i}} .
$$

From (3.11)

$$
\begin{aligned}
\frac{\partial \mathcal{L}_{m}}{\partial \phi^{l}} & =\frac{\partial\left(c_{2} e^{\alpha_{1} \sigma} \mathcal{M}_{I J} * F^{I} \wedge F^{J}\right)}{\partial \phi^{l}} \\
& =\frac{\partial\left(c_{2} e^{\alpha_{1} \sigma} \nu_{I}^{A} \nu_{J}^{A} * F^{I} \wedge F^{J}\right)}{\partial \phi^{l}} \\
& =c_{2} e^{\alpha_{1} \sigma}\left(\partial_{l} \nu_{I}^{A} \nu_{J}^{A}+\nu_{I}^{A} \partial_{l} \nu_{J}^{A}\right) * F^{I} \wedge F^{J} .
\end{aligned}
$$

Since in the parametrization (3.7) the dilatons $\phi^{i}$ are coupled to the Cartan 
generators $H_{i}$ which commute among themselves within the Cartan subalgebra structure of $o(10-D, 10-D+N)$ one can easily show that

$$
\frac{\partial \nu}{\partial \phi^{l}} \equiv \partial_{l} \nu=\frac{1}{2} H_{l} \nu
$$

Thus we have

$$
\partial_{l} \nu_{I}^{A}=\left(\partial_{l} \nu\right)_{I}^{A}=\left(\frac{1}{2} H_{l} \nu\right)_{I}^{A}=\frac{1}{2}\left(H_{l}\right)_{N}^{A} \nu_{I}^{N}
$$

Inserting this result back in (3.53) we obtain

$$
\frac{\partial \mathcal{L}_{m}}{\partial \phi^{l}}=c_{2} e^{\alpha_{1} \sigma}\left(\frac{1}{2}\left(H_{l}\right)_{N}^{A} \nu_{I}^{N} \nu_{J}^{A}+\frac{1}{2} \nu_{I}^{A}\left(H_{l}\right)_{N}^{A} \nu_{J}^{N}\right) * F^{I} \wedge F^{J}
$$

Now since both $F^{I}$ and $F^{J}$ are two-forms by using the identity

$$
\left(H_{l}\right)_{N}^{A} \nu_{I}^{N} \nu_{J}^{A} * F^{I} \wedge F^{J}=\left(H_{l}\right)_{N}^{A} \nu_{I}^{N} \nu_{J}^{A} * F^{J} \wedge F^{I}
$$

and by arranging the indices above we conclude that

$$
\begin{aligned}
\frac{\partial \mathcal{L}_{m}}{\partial \phi^{l}} & =c_{2} e^{\alpha_{1} \sigma} \nu_{I}^{A}\left(H_{l}\right)_{N}^{A} \nu_{J}^{N} * F^{I} \wedge F^{J} \\
& =c_{2} e^{\alpha_{1} \sigma} * F \wedge \nu^{T} H_{l} \nu F
\end{aligned}
$$

which justifies its existence in (3.51). In this section we have derived the set of equations (3.14), (3.16), (3.24) and (3.51) which are the field equations of the $O(p, q) / O(p) \times O(q)$ symmetric space sigma model coupled generally to a dilaton, a certain number of abelian gauge fields and a two-form which is coupled through its Chern-Simons field strength. We have shown that the equations (3.24) can be expressed more explicitly in terms of the coset scalars through the calculation of the Cartan-Maurer form. Thus we have also derived a simplified set of equations namely the equations (3.37) as alternatives of (3.24). The equations (3.50) are also written more compactly 
in (3.51). Before we link our results to the $D$-dimensional toroidally compactified heterotic string we will give three examples on which the results we have obtained are applicable.

\subsection{The $\mathrm{D}=7$ Case}

The bosonic lagrangian of the $\mathcal{N}=2, D=7$ Maxwell-Einstein supergravity is constructed in [31] as

$$
\begin{aligned}
\mathcal{L}= & \frac{1}{2} R * 1-\frac{5}{8} * d \sigma \wedge d \sigma-\frac{1}{2} e^{2 \sigma} * G \wedge G \\
& +\frac{1}{4} \operatorname{tr}\left(* d \mathcal{M}^{-1} \wedge d \mathcal{M}\right)-\frac{1}{2} e^{\sigma} F \wedge \mathcal{M} * F,
\end{aligned}
$$

where the coupling between the field strengths $F^{I}=d A^{I}$ for $I=1, \ldots, N+3$ and the scalars which parameterize the coset $S O(N, 3) / S O(N) \times S O(3)$ can be explicitly written as

$$
-\frac{1}{2} e^{\sigma} F \wedge \mathcal{M} * F=-\frac{1}{2} e^{\sigma} \mathcal{M}_{I J} F^{I} \wedge * F^{J} .
$$

In writing (3.59) we have assumed the $(N+3)$-dimensional matrix representation of $s o(N, 3)$. The Chern-Simons form $G$ is defined as [31]

$$
G=d B-\frac{1}{\sqrt{2}} \eta_{I J} A^{I} \wedge F^{J},
$$

where $\eta=\operatorname{diag}(-,-,-,+,+, \ldots,+)$ is the invariant tensor of $S O(N, 3)$. If we compare (3.59) with (3.13) we see that

$$
\begin{gathered}
c_{1}=-\frac{5}{8} \quad, \quad c_{2}=c_{3}=-\frac{1}{2}, \\
\alpha_{1}=1 \quad, \quad \alpha_{2}=2 .
\end{gathered}
$$


Also comparing (3.61) with (3.12) yields

$$
c_{4}=-\frac{1}{\sqrt{2}}
$$

Thus with the above identification of the coefficients (3.14), (3.16), (3.24) and (3.51) give us the bosonic field equations of the $\mathcal{N}=2, D=7$ MaxwellEinstein supergravity in a general solvable Lie algebra parametrization of the coset $S O(N, 3) / S O(N) \times S O(3)$. We should state that although this coset is different than the one we have assumed in (3.3) the formal, abstract construction of the lagrangian in the solvable Lie algebra gauge we have performed for (3.3) is also valid for $S O(N, 3) / S O(N) \times S O(3)$.

\subsection{The $\mathrm{D}=8$ Case}

The lagrangian of the bosonic sector of the $\mathcal{N}=1, D=8$ Maxwell-Einstein supergravity is given as 32

$$
\begin{aligned}
\mathcal{L}= & \frac{1}{4} R * 1+\frac{3}{8} * d \sigma \wedge d \sigma-\frac{1}{2} e^{2 \sigma} * G \wedge G \\
& +\frac{1}{4} \operatorname{tr}\left(* d \mathcal{M}^{-1} \wedge d \mathcal{M}\right)-\frac{1}{2} e^{\sigma} F \wedge \mathcal{M} * F .
\end{aligned}
$$

The Chern-Simons three-form $G$ is

$$
G=d B+\eta_{I J} F^{I} \wedge A^{J}
$$

with $I, J=1, \ldots, N+2$. Apart from the single dilaton the rest of the scalars parameterize the coset manifold $S O(N, 2) / S O(N) \times S O(2)$ and the $S O(N, 2)$ invariant tensor $\eta$ is $\eta=\operatorname{diag}(-,-,+,+, \ldots,+)$. We again assume that we choose an $(N+2)$-dimensional matrix representation of $s o(N, 2)$. Again if 
we compare (3.64) with (3.13) in this case we have

$$
\begin{gathered}
c_{1}=\frac{3}{8} \quad, \quad c_{2}=c_{3}=-\frac{1}{2}, \\
\alpha_{1}=1 \quad, \quad \alpha_{2}=2 .
\end{gathered}
$$

Also from (3.65) and (3.12) we have

$$
c_{4}=1
$$

If the coefficients are chosen as above then (3.14), (3.16), (3.24) and (3.51) correspond to the bosonic field equations of the $\mathcal{N}=1, D=8$ MaxwellEinstein supergravity in a general solvable Lie algebra parametrization of the coset $S O(N, 2) / S O(N) \times S O(2)$.

\subsection{The $\mathrm{D}=9$ Case}

As a final example we will consider the $\mathcal{N}=1, D=9$ Maxwell-Einstein supergravity [33]. The bosonic lagrangian of the $\mathcal{N}=1, D=9$ MaxwellEinstein supergravity can be given as 33.

$$
\begin{aligned}
\mathcal{L}= & -\frac{1}{4} R * 1+\frac{7}{4} * d \sigma \wedge d \sigma+\frac{1}{2} e^{-4 \sigma} * G \wedge G \\
& +\frac{1}{4} \operatorname{tr}\left(* d \mathcal{M}^{-1} \wedge d \mathcal{M}\right)-\frac{1}{2} e^{-2 \sigma} F \wedge \mathcal{M} * F .
\end{aligned}
$$

In this case the scalars of the coupling abelian vector multiplets parameterize the scalar coset $S O(N, 1) / S O(N)$. The Chern-Simons three-form is taken as

$$
G=d B+\eta_{I J} A^{I} \wedge F^{J}
$$


with $I, J=1, \ldots, N+1$. We assume an $(N+1)$-dimensional matrix representation of $s o(N, 1)$. Similar to the $D=7$ and $D=8$ cases we have $\eta=\operatorname{diag}(-,+,+, \ldots,+)$ which is the invariant tensor of $S O(N, 1)$. The comparison of (3.68) and (3.69) with (3.13) and (3.12) respectively specifies the coefficients as

$$
\begin{gathered}
c_{1}=\frac{7}{4} \quad, \quad c_{2}=-\frac{1}{2} \quad, \quad c_{3}=\frac{1}{2}, \\
\alpha_{1}=-2 \quad, \quad \alpha_{2}=-4 \quad, \quad c_{4}=1 .
\end{gathered}
$$

Therefore again with the above identification of the coefficients (3.14), (3.16), (3.24) and (3.51) become the bosonic field equations of the $\mathcal{N}=1, D=9$ Maxwell-Einstein supergravity for a general solvable Lie algebra parametrization of the coset $S O(N, 1) / S O(N)$. We should remark that the discussion we have made for the replacement of (3.3) with the coset $S O(N, 3) / S O(N) \times$ $S O(3)$ of the $D=7$ case is also valid for the $D=8 \operatorname{coset} S O(N, 2) / S O(N) \times$ $S O(2)$ and the $D=9 \operatorname{coset} S O(N, 1) / S O(N)$.

\section{Field Equations of the D-dimensional Het- erotic String}

In section two we have given the bosonic lagrangian of the toroidally compactified $E_{8} \times E_{8}$ low energy effective heterotic string. The Kaluza-Klein reduction produces the scalar manifolds which are in the form of (2.25). The scalar lagrangian is written as (2.22) by using the explicit representation (2.24) of the generators of the solvable Lie algebra of $o^{\prime}(10-D+N, 10-D)$ in the coset parametrization (2.23). As we have already mentioned before the importance of this formulation is that it is based on the Kaluza-Klein descendant fields. In section three however we have taken our scalar coset 
manifold explicitly as $O(10-D, 10-D+N) / O(10-D) \times O(10-D+N)$ whose definition originates from the indefinite signature metric (3.2). We have to remark two points now. Firstly in the definition of the generalized orthogonal groups $O(p, q)$ the indefinite signature metric can be replaced by any symmetric matrix which has $p$ positive and $q$ negative eigenvalues. The resulting group of matrices may differ from each other from their set content point of view however their group structures are all isomorphic to each other [30]. Also since $\operatorname{Spin}(p, q)$ is a double cover of $O(p, q)$ and since it is isomorphic to $\operatorname{Spin}(q, p)$ [35] we have the isomorphisms

$$
\begin{aligned}
& O^{\prime}(10-D+N, 10-D) \simeq O^{\prime}(10-D, 10-D+N) \\
& \simeq O(10-D+N, 10-D) \simeq O(10-D, 10-D+N) .
\end{aligned}
$$

Thus we observe that from the algebraic point of view the choice of the global symmetry group representation among (4.1) which defines the scalar coset manifold is unimportant. However the choice of $O^{\prime}(10-D+N, 10-D)$ is distinct since it arises naturally during the Kaluza-Klein reduction in [22]. On the other hand the choice of the global symmetry group representation effects the structure of the field equations via the indefinite metric which explicitly appears in the equations. The second point we should emphasize on is that the properties of $\eta$ which we have made use of to derive the bosonic field equations of the scalar coset with couplings in the previous section are all shared by $\Omega$. First of all as it can be deduced directly from (2.21)

$$
\Omega^{2}=1
$$

Also since $\Omega$ is a symmetric matrix and the coset representatives (2.23) of $O^{\prime}(10-D+N, 10-D) / O(10-D+N) \times O(10-D)$ satisfy (2.20) the identities (3.27) - (3.32) are shared by $\Omega$ and the coset representatives (2.23). Therefore we may conclude that we may replace $\eta$ by $\Omega$ in the formulation performed 
in the last section, thus our results can be extended to the Kaluza-Klein scalar coset representation of section two. In other words all the formulas of section three are legitimately correct when one replaces $\eta$ by $\Omega$ taking the scalar coset manifold as $O^{\prime}(10-D+N, 10-D) / O(10-D+N) \times O(10-D)$. One final remark we should do before writing down the field equations of the heterotic string is about the coset parametrization. Comparing the coset parametrizations (2.23) and (3.7) which are both based on the solvable Lie algebra of the global symmetry group one sees that one needs a transformation to relate the fields $\left\{\varphi^{i}, A_{(0) j}^{i}, A_{(0) i j}, B_{(0) i}^{I}\right\}$ to the ones $\left\{\phi^{i}, \chi^{m}\right\}$. Thus one should consider the equality of the two coset parametrizations

$$
e^{\frac{1}{2} \varphi^{i} H_{i}} e^{A_{(0) j}^{i} E_{i}^{j}} e^{\frac{1}{2} A_{(0) i j} V^{i j}} e^{B_{(0) i}^{I} U_{I}^{i}}=e^{\frac{1}{2} \phi^{i} H_{i}} e^{\chi^{m} E_{m}},
$$

bearing in mind that while the solvable Lie algebra generators on the left hand side are fixed by the representation chosen and by their embedding in $o^{\prime}(10-D+N, 10-D)$ which is discussed in [22], the solvable Lie algebra generators on the right hand side are completely arbitrary. The transformation can be calculated in two ways; either after choosing the basis on both sides of (4.3) the same, one considers local scalar fields whose ranges in the exponential generate solvable Lie algebra elements in a sufficiently small neighborhood of the identity element so that (4.3) is valid in any representation chosen for the algebra, thus giving the transformations explicitly as a matrix equation 2 or after determining another solvable Lie algebra $\mathbf{s}_{0}$ of $o^{\prime}(10-D+N, 10-D)$ through a selection of an Iwasawa decomposition on the right hand side of (4.3), one may identify the matrix representatives of any basis of this solvable Lie algebra $\left\{H_{i}, E_{m}\right\}$ in the representation generated by (2.24) then one can find the field transformations from (4.3) explicitly again. Despite the locality of the first method by following the second method one may find global field transformations which depend on

\footnotetext{
${ }^{2} \mathrm{~A}$ similar discussion leads to the differential form of such scalar field transformations in [29].
} 
the particular representation of section two. Therefore keeping in mind that one can always find such transformations between the scalar field definitions of the two different coset parametrizations as discussed above, in writing the field equations of the $D$-dimensional compactified heterotic string we will assume a coset parametrization in the form of (3.7) for the scalar coset manifold $O^{\prime}(10-D+N, 10-D) / O(10-D+N) \times O(10-D)$ which is the one used in the previous section. Comparing the lagrangians (2.26) and (3.13) we firstly observe that

$$
\eta \rightarrow \Omega
$$

Then

$$
\begin{gathered}
c_{1}=c_{2}=c_{3}=-\frac{1}{2} \\
\alpha_{1}=-\sqrt{\frac{2}{(D-2)}}, \quad \alpha_{2}=-\sqrt{\frac{8}{(D-2)}} .
\end{gathered}
$$

Also

$$
\begin{gathered}
\sigma \equiv \phi, \quad G \equiv F_{(3)}, \quad C_{(1)}^{I} \equiv A^{I}, \\
B \equiv A_{(2)}, \quad F^{I} \equiv H_{(2)}^{I} .
\end{gathered}
$$

Furthermore

$$
\begin{aligned}
F_{(3)} & =d A_{(2)}+\frac{1}{2} C_{(1)}^{T} \Omega d C_{(1)} \\
& =d A_{(2)}+\frac{1}{2} \Omega_{I J} C_{(1)}^{I} \wedge d C_{(1)}^{J},
\end{aligned}
$$


which is in the form of (3.12) with the identification of the coupling constant as

$$
c_{4}=\frac{1}{2}
$$

We may conclude that in the light of these identifications the formalism which appears in section two is in parallel with the general one constructed in section three differing only in the scalar coset representation. Finally upon the discussion we have made about the coset parametrizations, if we consider the field redefinitions and the substitutions mentioned above in the formalism of the previous section a similar line of derivation would give us the bosonic field equations of the $D$-dimensional toroidally compactified $E_{8} \times E_{8}$ low energy effective heterotic string as

$$
\begin{aligned}
(-1)^{D} d(* d \phi)= & \frac{1}{2} \sqrt{8 /(D-2)} e^{-\sqrt{\frac{8}{(D-2)}} \phi} * F_{(3)} \wedge F_{(3)} \\
& +\frac{1}{2} \sqrt{2 /(D-2)} e^{-\sqrt{\frac{2}{(D-2)}} \phi} \mathcal{M}_{I J} * H_{(2)}^{I} \wedge H_{(2)}^{J}, \\
d\left(e^{-\sqrt{\frac{8}{(D-2)}} \phi} * F_{(3)}\right)= & 0 \\
d\left(e^{-\sqrt{\frac{2}{(D-2)}} \phi} \mathcal{M} * H_{(2)}\right)= & (-1)^{D} e^{-\sqrt{\frac{8}{(D-2)}} \phi} \Omega H_{(2)} \wedge * F_{(3)}, \\
d\left(* d \phi^{i}\right)= & \frac{1}{2} \sum_{\beta \in \Delta_{n c}^{+}} \beta_{i} e^{\frac{1}{2} \beta_{j} \phi^{j}} U^{\beta} \wedge e^{\frac{1}{2} \beta_{j} \phi^{j}} * U^{\beta} \\
& -\frac{1}{2}(-1)^{D} e^{-\sqrt{\frac{2}{(D-2)}} \phi} * H_{(2)} \wedge \nu^{T} H_{i} \nu H_{(2)}, \\
d\left(e^{\gamma_{i} \phi^{i}} * U^{\gamma}\right)= & \sum_{\alpha-\beta=-\gamma} N_{\alpha,-\beta} U^{\alpha} \wedge e^{\beta_{i} \phi^{i}} * U^{\beta}
\end{aligned}
$$


where the coset representatives

$$
\nu=e^{\frac{1}{2} \phi^{i} H_{i}} e^{\chi^{m} E_{m}},
$$

satisfy the defining relation of $O^{\prime}(10-D+N, 10-D)$ namely

$$
\nu^{T} \Omega \nu=\Omega,
$$

and they parameterize the coset

$$
O^{\prime}(10-D+N, 10-D) / O(10-D+N) \times O(10-D),
$$

as we have discussed in detail above. Before concluding we will discuss one more point. One may investigate the relation between the two formulations considered in this section also in section two and the one introduced in section three which are constructed over different scalar coset representations although they are two equivalent formulations of the same theory. This is a matter of interest since when we take a look at (4.7) and (4.9) we see that as $\Omega$ is a non-diagonal matrix there is a degree of mixing of the abelian gauge fields and their field strengths in the Chern-Simons three-form (4.7). This mixing brings a certain complexity of coupling in the field equations (4.9). However in the formulation of section three this mixing is avoided since $\eta$ is a diagonal matrix. Thus the field equations obtained by using the scalar coset representation based on $\eta$ involve less coupling which may be a simplification and an advantage in seeking solutions. One may find solutions in the $\eta$-formulation and then pass to the Kaluza-Klein field content by using the field transformations upon the assumption that the lagrangians of these two distinct formulations are equal. This approach is different than the one used above which derives the field equations of the $D$-dimensional heterotic string solely in the $\Omega$-formulation. More specifically one would consider the equality of the two lagrangians which are based on the scalar 
coset manifolds of $O^{\prime}(10-D+N, 10-D) / O(10-D+N) \times O(10-D)$ and $O(10-D, 10-D+N) / O(10-D) \times O(10-D+N)$ respectively 3 . In this case one has to calculate the field transformations between the constructions based on two different scalar coset representations. We may calculate the transformations of the field contents by equating the two lagrangians (2.26) and (3.13) which are based on different scalar coset matrix structures. If we do so we see that all the previous coupling constant and field equivalences mentioned in this section are valid except the ones for $A^{I}$ and the coset scalars since in this case we have

$$
\frac{1}{2} \Omega_{I J} C_{(1)}^{I} \wedge d C_{(1)}^{J}=c_{4} \eta_{I J} A^{I} \wedge F^{J}
$$

and

$$
-\frac{1}{2} e^{-\sqrt{2 /(D-2)} \phi} * H_{(2)}^{T} \wedge \mathcal{M} H_{(2)}=c_{2} e^{\alpha_{1} \sigma} * F \wedge \mathcal{M} F .
$$

On the left hand side of the above equation the coset parametrization is (2.23) and on the right hand side it is (3.7). If one assumes a transformation between the gauge fields $C_{(1)}^{I}$ and $A^{I}$ of the two formulations in the form

$$
C_{(1)}=T A,
$$

where $T$ is a $(20-2 D+N) \times(20-2 D+N)$ matrix then by choosing $c_{4}=1 / 2$ again one finds that from (4.13) $T$ must satisfy

$$
T^{T} \Omega T=\eta .
$$

\footnotetext{
${ }^{3}$ Note that although we have used the first coset structure for the $D$-dimensional heterotic string in section two and four and the second one in our general formalism in section three we have made use of the results of section three for the $D$-dimensional heterotic string by simply substituting $\Omega$ instead of $\eta$ owing to the similar properties of these two metrics which take part in the derivation of the field equations. However we have not mentioned relating these two formulations before.
} 
Inserting (4.15) in (4.14) one also finds that

$$
\nu^{\prime}=\nu T
$$

Since $\Omega$ is a symmetric matrix and since any symmetric matrix can be diagonalized in the form (4.16) with $T \in O(20-2 D+N)$ [36, 37, 38] one can find the transformation matrix $T$ to relate the two different set of gauge fields. After determining the solvable Lie algebra $\mathbf{s}_{0}$ of $O(10-D, 10-D+N)$ through a selection of an Iwasawa decomposition (3.4) one may choose a $(20-2 D+N)$ dimensional matrix representation to express the basis $\left\{H_{i}, E_{m}\right\}$ and then from (4.17) one may also find the transformation rules of the coset scalars between the two scalar coset formulations. In this way one may solve the field equations in the $\eta$-formulation which are less coupled and then pass to the field content obtained by the direct Kaluza-Klein reduction.

Although this method may look tempting we should also point out that the transformations obtained in this way can not cover the entire solution space thus they may be highly restrictive. The reason for this lies in the process of equating the two lagrangians (2.26) and (3.13) and obtaining (4.17). We have stated before that although the groups are isomorphic the matrix contents of the sets $O^{\prime}(10-D+N, 10-D)$ and $O(10-D, 10-D+N)$ may be quite different for example

$$
\begin{aligned}
& \Omega \in O^{\prime}(10-D+N, 10-D), \quad \Omega \notin O(10-D, 10-D+N), \\
& \eta \in O(10-D, 10-D+N), \quad \eta \notin O^{\prime}(10-D+N, 10-D) .
\end{aligned}
$$

Thus the transformations (4.17) can exist only for the localized coset scalars which would lead to the coset representatives that lie in $O^{\prime}(10-D+N, 10-D)$ when the representatives in $O(10-D, 10-D+N)$ are translated by $T$ from the right. On the other hand due to the generalized formulation of section 
three one has the degree of freedom of defining $\left\{\phi^{i}, \chi^{m}\right\}$ from a rich choice of alternatives of the Iwasawa decomposition, the solvable Lie algebra, the basis $\left\{H_{i}, E_{m}\right\}$ and the matrix representations of the Lie algebra $o(10-D, 10-$ $D+N)$. Finally we may state that to justify the transformations (4.17) one should focus on matching the coset representative images of the solvable Lie algebra parametrizations of the two distinct scalar coset structures of section two and section three via the right-translation by $T$.

\section{Conclusion}

A review of the Kaluza-Klein dimensional reduction [22] of the bosonic sector of the ten dimensional $\mathcal{N}=1$ simple supergravity that is coupled to $N$ abelian gauge multiplets [8, 9] on the tori $T^{10-D}$ which describes the massless sector of the $E_{8} \times E_{8}$ heterotic string theory [14] is given in section two. Then we have derived the field equations of the general symmetric space sigma model with dilaton, abelian matter and Chern-Simons couplings in section three. In section four we have used the results of section three to express the bosonic field equations of the $D$-dimensional heterotic string whose bosonic lagrangian is in parallel with the general lagrangian we have considered in section three. Besides as we have discussed by the end of section three our general formulation corresponds to the one that occurs when the MaxwellEinstein supergravities are constructed by the use of the Noether's method [31, 32, 33]. We have also mentioned about the possible transformations between the two different scalar coset parametrizations of section two and section three which are both in the solvable Lie algebra gauge. Finally in section four we have discussed an alternative way of deriving the field equations of the compactified heterotic string whose field content directly arises from the $D=10$ heterotic string via the Kaluza-Klein ansatz [22]. By this method one can express the field equations of the $D$-dimensional heterotic string exactly as in section three whose scalar coset matrix representations 
are different from the ones used in section two. One can then search for the field transformations which originate from the equality of the two distinct lagrangians of section two and section three. The limitations associated with this method are also pointed out in the last section.

In [23] the dynamics of the symmetric space sigma model with only abelian gauge field couplings is studied under the solvable Lie algebra gauge. Therefore apart from deriving the bosonic matter field equations of the $D$ dimensional $E_{8} \times E_{8}$ heterotic string this work generalizes the formulation of [23] to include further dilaton and Chern-Simons couplings. Our formulation in section three is based on a generic global symmetry group $O(p, q)$ and we derive the field equations for a general solvable Lie algebra parametrization of the scalar coset without specifying neither the basis used nor the representation chosen as in [23]. From this point of view the field equations we have obtained are extensive in their coverage and they are purely in algebraic terms. On the other hand the major achievement of this work is the explicit derivation of the bosonic matter field equations of the $D$-dimensional toroidally compactified $E_{8} \times E_{8}$ heterotic string for a generic and unspecified solvable Lie algebra parametrization of the scalar coset. Although the bosonic lagrangian constructed in [22] under the solvable Lie algebra gauge assumes a certain representation of the coset generators we have obtained the bosonic matter field equations of the $D$-dimensional heterotic string in a representation free formalism. Therefore for the field equations obtained in the last section we have a degree of freedom of choosing an appropriate representation of the solvable Lie algebra. This would provide a useful machinery for seeking solutions of the theory. As a matter of fact one can inspect various representations to simplify the field equations bearing in mind that choosing a basis for the solvable Lie algebra also corresponds to defining the coset scalar fields. Appropriate representations would decrease the complexity of the field equations. When one finds a suitable representation step by step one can construct the explicit equations of motion which would involve 
less couplings. If the field equations in section three and section four are inspected carefully one can see that the most essential coupling is between the coset scalars and the gauge fields beside the non-linearity of the structure of the coset scalars themselves. Thus focusing on these couplings we have also derived various versions of the corresponding field equations in section three which may provide a set of tools when certain solutions of these equations are studied.

In section four we have discussed that the formulation of section three which is based on the invariant metric $\eta$ is advantageous over the formulation descending from the Kaluza-Klein compactification so that $\eta$ is a diagonal matrix and it prevents cross couplings of the gauge fields in the field equations. Since our aim in this work is to formulate the field equations rather than studying the solutions we have not given specific examples for which this simplicity may be exploited. However we may state that the $\eta$ formulation brings simplicity in deriving the first-order field equations of the Maxwell-Einstein supergravities and in constructing the non-linear realizations of these theories [39, 40, 41]. For the $\eta$-formulation from [39, 40, 41] we observe that the non-mixing of the gauge fields in the field equations is reflected in the coset algebra of the non-linear sigma model construction of the theory such that the operators which correspond to different gauge fields do not mix under the algebra product (they anti-commute with each other).

Although we have mentioned about the relation between the two different solvable Lie algebra parametrizations of the scalar coset in the last section we have not shown any attempt to construct the transformations explicitly. As we have discussed one can search for the local or the global scalar field transformations which would make use of the group theoretical structure of the global symmetry group. One can also inspect the field transformations mentioned in the last part of section four which relate the field contents of the two equivalent formulations of the theory based on two distinct scalar coset representations. As we have stated before this would remove some 
complexity of the field equations. Therefore one can workout the solutions in the formulation of section three and then transform the fields to the original Kaluza-Klein field content of section two.

The formalism of section three can be extended for theories which posses different global symmetry groups than $O(p, q)$ but which still can pertain the solvable Lie algebra gauge. In this case the scalar coset $G / K$ must be such that again the global symmetry group $G$ must be a real form of a non-compact semi-simple Lie group and $K$ must be its maximal compact subgroup to be able to apply the solvable Lie algebra gauge to parameterize the scalar coset manifold [18]. However when defining the internal metric one must use a generalized transpose which is induced by the Cartan involution of the Lie algebra $g$ [18, 23, 26, 27, 28, 29] instead of the ordinary matrix transpose. Since the supergravity theories give way to first-order formulations [19, 20, 42] the first-order formulation of the field equations can be performed for both the general formalism of section three and the $D$-dimensional compactified heterotic string. Finally we should state that although owing to the non-linear structure of the coset scalars also the complexity of the coupling between the scalars and the gauge fields we have focussed on the bosonic matter sector in this work one can extend the solvable Lie algebra gauge formalism developed here to derive the field equations in the presence of the graviton and the fermionic sectors too.

\section{References}

[1] E. Cremmer, B. Julia and J. Scherk, "Supergravity theory in elevendimensions", Phys. Lett. B76 (1978) 409.

[2] I. C. G. Campbell and P. C. West, "N=2, $d=10$ non-chiral supergravity and its spontaneous compactification", Nucl. Phys. B243 (1984) 112. 
[3] M. Huq and M. A. Namazie, "Kaluza-Klein supergravity in tendimensions", Class. Quant. Grav. 2 (1985) 293.

[4] F. Giani and M. Pernici, "N=2 supergravity in ten-dimensions", Phys. Rev. D30 (1984) 325.

[5] J. H. Schwarz and P. C. West, "Symmetries and transformations of chiral $N=2$ d=10 supergravity", Phys. Lett. B126 (1983) 301.

[6] P. S. Howe and P. C. West, "The complete N=2, d=10 supergravity", Nucl. Phys. B238 (1984) 181.

[7] J. H. Schwarz, "Covariant field equations of chiral $N=2$ d=10 supergravity", Nucl. Phys. B226 (1983) 269.

[8] E. Bergshoeff, M. de Roo, B. de Wit and P. van Nieuwenhuizen, "Tendimensional Maxwell-Einstein supergravity, its currents, and the issue of its auxiliary fields", Nucl. Phys. B195 (1982) 97.

[9] G. F. Chapline and N. S. Manton, "Unification of Yang-Mills theory and supergravity in ten-dimensions", Phys. Lett. B120 (1983) 105.

[10] P. C. West, "Introduction to Supersymmetry and Supergravity", (2nd Edition, World Scientific Pub. Co. 1990).

[11] A. Salam (Ed.), E. Sezgin (Ed.), "Supergravities in Diverse Dimensions", (World Scientific Pub. Co. 1989).

[12] P. C. West, "Supergravity, brane dynamics and string duality", hep-th/9811101.

[13] Y. Tanii, "Introduction to supergravities in diverse dimensions", hep-th/9802138.

[14] E. Kiritsis, "Introduction to superstring theory", hep-th/9709062. 
[15] C. M. Hull and P. K. Townsend, "Unity of superstring dualities", Nucl. Phys. B438 (1995) 109, hep-th/9410167.

[16] E. Witten, "String theory dynamics in various dimensions", Nucl. Phys. B443 (1995) 85, hep-th/9503124.

[17] L. Andrianopoli, R. D'Auria, S. Ferrara, P. Fre, M. Trigiante, " $R-R$ scalars, U-duality and solvable Lie algebras", Nucl. Phys. B496 (1997) 617, hep-th/9611014.

[18] S. Helgason, "Differential Geometry, Lie Groups and Symmetric Spaces", (Graduate Studies in Mathematics 34, American Mathematical Society Providence R.I. 2001).

[19] E. Cremmer, B. Julia, H. Lü and C. N. Pope, "Dualisation of Dualities. I.", Nucl. Phys. B523 (1998) 73, hep-th/9710119.

[20] E. Cremmer, B. Julia, H. Lü and C. N. Pope, "Dualisation of dualities II : Twisted self-duality of doubled fields and superdualities", Nucl. Phys. B535 (1998) 242, hep-th/9806106.

[21] C. N. Pope, "Lecture Notes on Kaluza-Klein Theory", (unpublished).

[22] H. Lü, C. N. Pope and K. S. Stelle, "M-theory/heterotic duality: A Kaluza-Klein perspective", Nucl. Phys. B548 (1999) 87, hep-th/9810159.

[23] T. Dereli and N. T. Yllmaz, "Dualisation of the symmetric space sigma model with couplings", Nucl. Phys. B705 (2005) 60, hep-th/0507007.

[24] H. Lü and C. N. Pope, "p-brane solitons in maximal supergravities", Nucl. Phys. B465 (1996) 127, hep-th/9512012. 
[25] E. Bergshoeff, D. C. Jong and E. Sezgin, "Noncompact gaugings, chiral reduction and dual sigma models in supergravity", Class. Quant. Grav. 23 (2006) 2803, hep-th/0509203.

[26] A. Keurentjes, "The group theory of oxidation", Nucl. Phys. B658 (2003) 303, hep-th/0210178.

[27] A. Keurentjes, "The group theory of oxidation II : Cosets of non-split groups", Nucl. Phys. B658 (2003) 348, hep-th/0212024.

[28] N. T. Yllmaz, "Dualisation of the general scalar coset in supergravity theories", Nucl. Phys. B664 (2003) 357, hep-th/0301236.

[29] N. T. Yllmaz, "The non-split scalar coset in supergravity theories", Nucl. Phys. B675 (2003) 122, hep-th/0407006.

[30] A. W. Knapp, "Lie Groups Beyond an Introduction", (Progress in Mathematics vol. 140, 2nd Edition, Birkhauser, Boston 2002).

[31] E. Bergshoeff, I. G. Koh, E. Sezgin, "Yang-Mills/Einstein supergravity in seven-dimensions", Phys. Rev. D32 (1985) 1353.

[32] A. Salam and E. Sezgin, "D = 8 supergravity: Matter couplings, gauging and Minkowski compactification", Phys. Lett. B154 (1985) 37.

[33] S. J. Gates, H. Nishino and E. Sezgin, "Supergravity in $d=9$ and its coupling to non-compact $\sigma$ model", Class. Quant. Grav. 3 (1986) 21.

[34] W. E. Thirring, "A Course in Mathematical Physics I and II: Classical Dynamical Systems and Classical Field Theory", (Springer-Verlag Berlin and Heidelberg GmbH and Co. KG 1991).

[35] P. Lounesto, "Clifford Algebras and Spinors", (London Mathematical Society Lecture Note Series 286, 2nd Edition, Cambridge University Press 2001). 
[36] K. M. Hoffman, R. A. Kunze, "Linear Algebra", (2nd Edition, Prentice Hall 1971).

[37] B. Schutz, "Geometrical Methods of Mathematical Physics", (Cambridge University Press 1980).

[38] J. F. Cornwell, "Group Theory in Physics: An introduction", (Academic Press 1997).

[39] N. T. Yllmaz, "Dualisation of the $d=7$ heterotic string", JHEP 0409 (2004) 003, hep-th/0507008.

[40] T. Dereli and N. T. Yllmaz, "Dualisation of the Salam-Sezgin d=8 supergravity", Nucl. Phys. B691 (2004) 223, hep-th/0407004.

[41] N. T. Yllmaz, "Dualisation of the $d=9$ matter coupled supergravity", JHEP 0506 (2005) 031, hep-th/0507011.

[42] B. Julia, "Superdualities: Below and beyond U-duality", hep-th/0002035. 